\title{
Métissages dans les productions circassiennes et chorégraphiques contemporaines
}

\section{Betty Lefèvre et Magali Sizorn}

\section{(2) OpenEdition}

1 Journals

Édition électronique

URL : http://journals.openedition.org/corpsetculture/800

DOI : 10.4000/corpsetculture.800

ISSN : $1777-5337$

Éditeur

Association Corps et Culture

Édition imprimée

Date de publication : 1 janvier 2004

ISSN : 1268-5631

Référence électronique

Betty Lefèvre et Magali Sizorn, « Métissages dans les productions circassiennes et chorégraphiques contemporaines », Corps et culture [En ligne], Numéro 6/7 | 2004, mis en ligne le 11 octobre 2007. consulté le 08 septembre 2020. URL : http://journals.openedition.org/corpsetculture/800 ; DOI https://doi.org/10.4000/corpsetculture.800

Ce document a été généré automatiquement le 8 septembre 2020

(c) tous droits réservés 


\title{
Métissages dans les productions circassiennes et chorégraphiques contemporaines
}

\author{
Betty Lefèvre et Magali Sizorn
}

1 Il est aujourd'hui banal de rappeler que la danse contemporaine, comme le cirque et la grande majorité des arts du vivant, offre des espaces de partage, de décloisonnements disciplinaires et identitaires. En outre, il semblerait que la population française témoigne d'un regain d'intérêt pour des formes artistiques "populaires » et « urbaines » : une enquête du Ministère de la culture de 1997 (Forette D., 1998), tend à montrer un engouement, tous milieux sociaux confondus, pour les représentations des «nouveaux » cirques .

2 Comment interpréter ces constatations? Par quelle alchimie mystérieuse ces formes artistiques potentialisent-elles les mouvances, les hésitations, les «surplus» de l'existence du social? Comment y invente-t-on de l'inédit, de l'altérité, de l'étrangeté ? Quels sont les modèles du corps, les déclinaisons identitaires, les valeurs à l'œuvre dans les créations chorégraphiques et circassiennes? Comment s'y manifeste notre présence charnelle et sensible au monde? Peut-on avancer que ces productions artistiques instaurent un nouvel « ordre » corporel, celui du métissage, en proposant des mises en chair transgressives et poétiques à partir desquelles se pensent d'autres réalités? En d'autres termes, comment l'expérience dansée ou circassienne fait-elle sens ? Comment ces mises en scène du corps s'inscrivent-elles dans le monde social et fondent, en retour, du lien social?

3 Ces arts revisités constituent un objet de recherche complexe, et même un défi épistémologique pour le sociologue : comment faire l'étude sociale des comportements esthétiques, voire des émotions, ces «formes sensibles de la vie sociale» dont parle Pierre Sansot (1986)?

Cadre theorique et methodologie

4 Penser la danse contemporaine et les arts du cirque, ce n'est pas seulement interroger des usages du corps particuliers, c'est aussi questionner l'expérience esthétique comme 
partage de l'éprouvé, du vécu, du sensible, en repérant comment ce cortège de sensations nourrit et se nourrit d'un rapport au monde.

5 C'est pourquoi le propos s'appuiera à la fois sur l'armature conceptuelle d'une sociologie compréhensive (au sens wébérien du terme, en considérant les productions artistiques comme des activités sociales chargées de sens aussi bien pour ceux qui en sont les acteurs que pour ceux qui en sont les spectateurs) et d'une phénoménologie vécue.

6 En effet, la danse contemporaine a jalonné nos vies, comme danseuses, chorégraphes, enseignantes, spectatrices, chercheures. Il nous semble disposer d'une co-naissance sur ce paysage de la danse contemporaine qui ne s'épuise pas dans la seule réalité de la chose dansée, mais qui se construit dans cette ballade au creux des expériences du corps, le notre, celui des autres. Et c'est cette coexistence, cette " réciprocité d'action » pour le dire avec Georges Simmel, qu'il s'agit ici de questionner (Simmel G., 1981 : 122). Plus récemment, les créations circassiennes ont élargi encore notre répertoire de curiosité.

7 La méthode employée emprunte à l'enquête ethnologique pour tenter de saisir au plus prêt, dans le détail des subjectivités, à la fois un terrain et le paysage évolutif de notre « être ensemble ».

8 Le recueil des données s'est fait par une étude méticuleuse de ce qui se donne à voir, et ce sont les spectacles proposés, les "cérémonies" (Beaud S., Weber F., 1997) circassiennes et chorégraphiques qui ont fait l'objet de nos observations. Ce travail d'investigation consiste à assister aux représentations et à repérer des relations, des codes, des normes culturelles comme autant d'éléments susceptibles de rendre compte «scientifiquement» de nos mises en scène sociales. D'autre part, les observations ont été complétées par l'aide précieuse d'informateurs privilégiés, de conversations informelles et d'entretiens semi-directifs avec des artistes contemporains.

9 Les réponses élaborées se feront à partir d'un échantillon de productions « récentes " de créateurs connus et dont on pourrait dire qu'ils représentent une certaine image de la danse et du cirque contemporains. Bien sûr, ce choix est arbitraire et illustre encore ce qui se noue entre le subjectif et l'objectif.

10 Notre hypothèse de travail s'attachera à démontrer que la danse et le cirque contemporains, entre autres, avancent d'autres référents où l'imaginaire social renoue avec une certaine effervescence dionysiaque, une esthétique de «l'être ensemble » néobaroque, et qui se montre dans l'exposition de formes hétéroclites : des formes difformes. De l'art métis

11 Les images proposées par ces productions contemporaines redéfinissent l'affirmation $\mathrm{du}$ corps comme expérience sensible et proposent la transformation d'un corps habitude en corps habité, d'un corps silence en corps parole, pour dire le monde et notre humanité, d'un corps ordinaire en corps extraordinaire. C'est ce que confirme Daniel Sibony lorsqu'il écrit : «La valeur d'une représentation est de susciter en nous, spectateurs ou acteurs ce sens de l'irreprésentable, c'est-à-dire de l'être ; bien qu'elle échoue à l'atteindre. Elle nous donne sa limite et ce n'est pas rien... Un bon spectacle, c'est le regardêtre qui nous émeut grâce au mouvement des corps vivants sous nos yeux. » (Sibony D., 1996 : 46-47).

12 Dans le cirque contemporain, les artistes vont au-delà d'une démonstration technique : il ne s'agit plus de léguer de génération en génération des "savoir-faire », mais des 
«savoir-être » en piste. Avec un discours nouveau, la narration devient l'élément constitutif des créations contemporaines. Polyvalents (danseurs, acrobates, musiciens, jongleurs, acteurs), ils incarnent des personnages et diffusent des messages, et ce métissage des arts a enrichi les productions circassiennes. Jean-Michel Guy précise que le cirque peut être considéré comme un "art dramatique ", car "capable de produire des textes (fussent-ils non écrits), d'élaborer du sens, en somme, de représenter » (Guy J.-M., 1998 : 35). C'est un cirque de créateurs formés aux techniques de cirque et à leur dépassement.

De la même façon, le corps acteur est au centre du propos de la danse contemporaine. Le corps n'est plus uniquement objet normé et passif du social, il se met en scène en agissant sur l'articulation entre des principes dialogiques.

14 Ainsi, chez Sasha Waltz, ce qui est mis à l'épreuve, c'est à la fois les corps en mouvement, mais surtout cette "marge " du geste où nos repères vacillent, où notre fascination pour le rationnel renoue avec l'irrationnel, un espace non visible où s'expriment un hors norme, un hors mesure, un hors la loi du moi, bref quelque chose aux confins du sacré et de la banalité d'une existence ordinaire. La pièce L'allée der kosmonauten (mais également la précédente Zweiland) nous invite à une déambulation identique à celle que l'on peut faire dans une foire à tout: un bric à brac de propositions, une décomposition des relations entre les êtres et entre les choses, des unions insolites, des dégradations d'où, curieusement, émerge une oscillation entre tragique et comique. Comme illustration, on peut évoquer cette planche qui danse entre les bouches de deux danseurs et qui devient étagère, lien ou obstacle, lieu de refuge ou prétexte à la violence. Cet objet ordinaire participe à cette histoire de famille, où trois générations se rencontrent, s'affrontent, s'aiment, combattent et jouent le jeu de notre réalité revisitée par d'autres corps.

Le métissage des arts est aujourd'hui largement répandu, voire généralisé. Il devient une source de créativité, et d'une certaine façon l'essence de cette exposition contemporaine des corps. "Arts métis » (Lachaud J.-M., 2001 : 139), danse et cirque constituent un espace d'échange, échange entre les arts, les techniques, les artistes.

Si les cirques traditionnels sont rassemblés autour de grands noms (les "grandes familles du cirque»), les compagnies de cirque contemporain sont les fruits de rencontres d'artistes différents réunis autour d'une production commune. Prenons l'exemple de la compagnie Lunatic: dans Petites histoires en l'air, quatre artistes, deux hommes et deux femmes, deux musiciens et deux aériens, développent un propos musical et corporel, sur les relations homme-femme.

Ce métissage artistique, que nous retrouvons également dans ChiencrU de Cahin-Caha et dans S.O.Y. du Kubilaï Khan Investigations, témoigne du vitalisme prolifique du cirque contemporain. De ces échanges artistiques découle une grande variété de productions, issues de ce que Gilbert Durand qualifie de "puissance esthétique de l'altérité " (Durand G., 1989 : 23). Chaque rencontre devient l'occasion d'une création singulière, où la personnalité même de l'artiste est investie.

Une esthétique à l'épreuve de l'altérité

Ces productions contemporaines nous invitent à un jeu avec la «multidimensionnalité et la polysémie de l'être social du corps » (Berthelot M., 1983). En d'autres termes, quels sont les imaginaires de l'altérité et de l'autre en soi à l'œuvre dans le cirque et la danse contemporaine? 

"retour du baroque» (Scarpetta G., 1988) dans la création artistique contemporaine et peut-être plus largement dans la société occidentale. Ainsi, et comme le souligne Jean Duvignaud, « (...) le baroque jette des formes qui tentent d'explorer métaphoriquement, par la fiction d'une psychologie éclatée, une réalité qu'elle n'appréhende pas. 
Eclatement de la vraisemblance qui corrompt les évidences et les représentations conventionnelles ou codées » (Duvignaud J., $1980: 111$ ).

Le cirque contemporain, créateur d'images nouvelles devient le théâtre d'expressions personnelles, où les circassiennes s'émancipent de l'ordre institué et transmis dans le cirque traditionnel, questionnant et récusant une définition univoque de la féminité et de son rapport au masculin.

De même, en danse contemporaine, le corps convoqué n'obéit plus à l'injonction d'un corps classique idéal, maîtrisé et triomphant, sorte de marionnette virtuose, mais il se fait l'écho de nos interrogations contemporaines sur le masculin-féminin, les souffrances de la sexualité, la vie/la mort, le sérieux et la drôlerie, l'indifférence et la différence. On pourrait résumer en écrivant qu'il s'agit de donner à voir des corps en action, des corps vécus avec une autonomie de sens et qui nous parlent du danseur acteur, du danseur chorégraphe, du danseur interprète, du danseur jongleur, du danseur musicien (c'est, par exemple, ce dialogue entre la danse et la musique qui fonde depuis 1983 le travail de la chorégraphe belge Anne Teresa de Keersmaeker).

La danse contemporaine fait varier nos mises en scène identitaires en autorisant l'expression des valeurs antagonistes qui nous fondent. Le danseur peut alors expérimenter l'Autre en soi en fouillant dans la " pluralité de ses masques » (Caillois R., 1950) pour mettre en scène l'ambiguïté et la pluralité des êtres qui nous habitent: l'androgyne (être ni homme ni femme), l'hermaphrodite (être homme et femme à la fois), le monstre (mi-homme, mi-bête), le démoniaque (mi-dieu, mi-homme).

C'est le résultat obtenu entre autre, par le chorégraphe italo-flamand Emio Gréco et sa partenaire dans une pièce intitulée Extra Dry: crâne rasé, seconde peau plissée par l'union de la sueur et d'une robe blanche transparente, corps androgyne à l'épreuve d'une synchronisation jamais atteinte, tous deux avec force et fragilité nous invitent au dépassement et/ou au dévoilement du codage identitaire. Jusqu'à l'épuisement, les danseurs déclinent une gestuelle à l'identique, sensuelle bien qu'ils ne se touchent jamais, de plus en plus irrépressible, proche de l'extase. A la fin du spectacle, la sueur a sculpté le tissu sur les corps, exposant, de face, la différence des sexes et, de dos, façonnant des écailles étrangement animales, reptiliennes, comme si l'identique créait le différent, comme si l'humain engendrait l'animal pour dire l'histoire toujours renouvelée de nos « rebroussements ».

Ce que nous avons pu observer dans les spectacles de danse contemporaine ou de nouveau cirque, ce n'est pas la séparation mais la conjonction, le « et + et + » des identifications multiples, une sorte d'errance d'avant le temps de la différence. Cette hybridation ou plutôt cette marqueterie identitaire, en substituant à la cohérence de l'Un la cohérence du Multiple, construit une invitation à expérimenter, comme l'écrit Michel Maffesoli « une communauté émotionnelle dans laquelle chacun simultanément se voile et se dévoile » (Maffesoli M., 1988).

Le métissage entre universalisme et particularisme culturels

Reconnu par l'U.N.E.S.C.O. comme un "art universel »" le cirque apparaît, et nous le dirons avec les mots de l'anthropologie, constitutif d'un "mythe fondateur " d'un imaginaire collectif mondial. Cependant, nous avons pu repérer au cours de nos observations des particularismes culturels. En effet, à travers le regard porté sur la place des femmes et leurs corporéités (Mauss M., 1950), nous avons constaté que des spectacles comme ceux du cirque national de Cuba ou de Circus Baobab (troupe nationale d'Art Acrobatique de Guinée) disent quelque chose de la culture de ces pays, 
et en particulier ici de la place qu'ils accordent à la femme. Dans le cirque national de Cuba, les femmes sont sensuelles, dénudées comme les danseuses de salsa. Dans La légende du singe tambourinaire de Circus Baobab, les femmes exécutent des danses traditionnelles africaines, danses de terre, et invitent le spectateur à partager ce moment, au rythme des djembés. Ces images de femmes diffèrent de celle de Cécile Mont-Reynaud de la compagnie Lunatic qui exprime, elle, les doutes et incertitudes identitaires des occidentales.

31 Le cirque apparaît donc comme le reflet de disparités culturelles : « toutes les identités culturelles ont leur cirque» (Bordes-Triballat A., 2001). Cette diversité, source de richesse, est également réinvestie au sein même de spectacles (comme ceux d'Arlette Gruss, de Pinder...), où des artistes de différentes nationalités présentent leurs spécialités. Ce sont les acrobaties chinoises, les contorsions mongoles ou encore la barre russe. Ces mélanges hétérogènes sont fréquents dans le cirque traditionnel, où l'exotisme est séduisant, et l'extraordinaire venu d'ailleurs.

Les nouvelles formes de productions circassiennes semblent perpétuer cette tradition d'ouverture. Néanmoins, il ne s'agit plus de juxtaposer des numéros disparates, mais de croiser les cultures. C'est alors parfois d'une rencontre et d'un échange que naissent l'extraordinaire, la surprise, l'émotion. Circus Baobab, parrainé par Pierrot Bidon, (fondateur d'Archaos) l'illustre en mêlant traditions africaines et occidentales : des trapézistes volent de branche en branche dans un baobab gigantesque, au-dessus de danseuses traditionnelles, acrobates et musiciens. Autre exemple de ce métissage dynamisant l'acte de création, Triptik, dernière production de Zingaro, dans laquelle Bartabas « rassemble Stravinsky, Boulez, des danseurs indiens, des jeunes femmes, des espagnols bais cerise, des lusitaniens crème aux yeux bleus et des sculptures équines de Jean-Louis Sauvat $»^{5}$.

Conclusion

33 Les arts du vivant que sont le cirque et la danse contemporaine décomposent nos habitudes de perception esthétique et recomposent notre regard à partir d'autres images comme celle de la dégradation (au sens d'un dégradé de couleur) et de la pluralité des formes, ce que nous avons appelé l'art du difforme. Dans cet imaginaire du hors cadre, tous les possibles sont investis, comme l'illustre la cantatrice de la Symphonie du Hanneton. Evadée de la toile qui la représentait en nature morte, elle se fait chimère : agrégat d'objets, de matières, de sons, de formes, de corps, elle devient femme-orchestre, femme-locomotive, symbole de cet imaginaire de la difformité.

Loin d'un simple jeu de convenance culturelle ou de mode marchande, ces spectacles mettent en scène la réflexion des arts et, pour le dire autrement, ce processus déjà évoqué en d'autres temps par Cicéron à propos de la culture latine, de « contamination » entre le théâtre, la musique, la danse, le cinéma, les arts du cirque, les arts plastiques.

Certains s'attachent au repérage d'un métissage culturel fécond, d'autres s'en inquiètent et développent l'idée d'un universalisme dévastateur des identités. En effet, l'emploi du terme métissage, emprunté à l'anthropologie du XIX ${ }^{\mathrm{e}}$ siècle, comporte le risque d'une interprétation péjorative qui se développerait autour d'une comparaison entre un art premier, pur, authentique et un art second, impur, bâtard. C'est ce qui anime souvent les débats autour d'un cirque traditionnel et le «nouveau » cirque, le cinéma et la nouvelle vague, la danse académique et la danse contemporaine, etc. Le métissage artistique est alors pensé comme une dégénérescence culturelle et devient le 
terrain possible d'expression d'une forme de refus de l'Autre, d'un racisme discret et nostalgique de l'Art avec un grand A.

Or, comme le rappelle Jean Duvignaud, l'histoire de l'homme est un immense partage et si toute culture vivante est un «bricolage" avec les éléments les plus divers (Duvignaud J., 1973), dans le domaine de la création artistique aussi il s'agit de bricoler pour donner à voir des formes anomiques, subversives, porteuses de ruptures, où mélange et impureté sont revendiqués comme l'expression d'un imaginaire sans limite.

En paraphrasant la réflexion de l'équipe de la revue Art Press (1987 : 31), on peut avancer que la danse, comme le cirque, est aujourd'hui l'inverse d'un art pur : « un extraordinaire foyer d'intégration, un empilement de contenus variés en natures, en propositions, ne correspondant sûrement plus à la «spécificité chorégraphique ». La danse et le cirque servent à recycler l'imaginaire et les savoirs [...]. Parce qu'ils ne rencontrent pas les autres langages, mais qu'ils les visitent, les danseurs font surgir entre les communautés artistiques des rapports d'un type nouveau, qui ne relèvent pas de la simple collaboration, mais de la réflexion d'un art sur un autre, réflexion d'où surgit l'événement. »

\section{BIBLIOGRAPHIE}

Art Press, « Les années danse » (1987), Hors série nº.

Beaud S., Weber F. (1997) Le Guide de l'enquête de terrain, Paris, La Découverte.

Berthelot J.M. (1983) Corps et société. Problèmes méthodologiques posés par une approche sociologique du corps, in Cahiers internationaux de sociologie, vol.LXXIV, 119-131.

Bordes-Triballat A. (2001) Les arts du cirque, une nouvelle pratique à l'école (partie 2), in Hyper E.P.S., 213, 18-27.

Durand G. (1989) Beaux-arts et Archétypes, Paris, PUF.

Duvignaud J. (1973) Les Ombres collectives. Sociologie du théâtre, Paris, PUF.

Duvignaud J. (1980) Le Jeu du jeu, Paris, Balland.

Forette D. (1998) Les arts de la piste : une activité fragile entre tradition et innovation, Rapport du conseil Economique et Social, $\mathrm{n}^{\circ} 15$.

Gautier F. (1999) Processus de différenciation sexuelle des corps dans les chorégraphies contemporaines, in actes du colloque La danse, une culture en mouvement, Strasbourg, Université Marc Bloch, 347-357.

Goffman E. (1977) La ritualisation de la féminité, in Les Moments et leurs hommes, Paris, Seuil/ Minuit, 150-185 (1988).

Guy J.-M. (1998) La transfiguration du cirque, in Théâtre aujourd'hui, 7, 26-51.

Hotier H. (1995) Cirque, Communication, Culture, PUB. 
Jung C. G., Kerenyi C. (1953) Introduction à l'essence de la mythologie, Paris, Payot et Rivages (2001).

Lacahud J.-M. (2001) Le cirque contemporain, entre collage et métissage in Guy J.-M. (dir.)

Autrement, Avant garde, Cirque!, 209, 126-141.

Mafffesoli M. (1988) Le Temps des tribus. Le déclin de l'individualisme dans les sociétés de masse, Paris, Pocket.

Mauss M. (1993 [1950]) Sociologie et anthropologie, Paris, PUF.

Sansot P. (1986) Les Formes sensibles de la vie sociale, Paris, PUF.

Scarpetta G. (1988) L’Artifice, Paris, Grasset.

Sibony D. (1996) Spectateur, spectateur, in Du théâtre, "La position de spectateur », hors série n5.

Simmel G. (1988) Philosophie de l'amour, Paris, Rivages.

Weber M. (1965) Essai sur la théorie de la science, Paris, Plon.

\section{NOTES}

1. Lors d'un entretien, Jean-Michel Guy nous avait fait remarquer que : " Ce qui est très clair chez les jongleuses, et je pense qu'on l'observe chez beaucoup de femmes ailleurs, c'est que la définition même de ce qu'est être une femme, appelons ça la féminité si vous voulez, est au cœur même de leur pratique, alors que ce n'est pas vrai des hommes $»$.

2. Selon Georges Simmel, « l'androgynisation exprime la réconciliation, la solidarité immédiate, organique, entre la personne et chacune de ses manifestations » (Simmel G., $1988: 74)$.

3. Nous le définirions avec C.G. Jung comme la réunion du masculin et du féminin (Jung C.G., Kerenyi C., 2001 [1953]).

4. Edouard Glissant, rédacteur en chef du numéro « le cirque, un art universel » (Le Courrier de l'U.N.E.S.C.O., Paris, janvier, 1988), précise que cette universalité est liée à trois éléments principaux : les disciplines et aspects lyriques et dramatiques du cirque qui ont inspiré d'autres arts (peinture, littérature...), son caractère international (avec les « gens du voyage »), et enfin, son caractère fraternel, lié au partage du sensible, du rire à la peur.

5. Garcin J., « Bartabas, Noir désir », Le nouvel observateur, $\mathrm{n}^{\circ} 1881,23-29$ novembre 2000, p. 8.

\section{RÉSUMÉS}

Les représentations contemporaines du corps en mouvement ne peuvent pas laisser le sociologue indifférent dès lors qu'il cherche à comprendre ce qui se joue entre l'acteur et le spectateur. Dans ce rapport d'interaction complexe, quel sens les créations chorégraphiques et circassiennes 
contemporaines prennent-elles pour le regardeur comme pour le regardé ? Quelles sont les figures symboliques à l'œuvre, des figures souvent contradictoires mais profondément efficaces pour réfléchir à la fois ce que le social inscrit sur les corps mais aussi ce qui se dévoile dans l'expression ludique avec les limites, avec l'Autre, avec ce qui dépasse le cadre?

La problématique du métissage est une façon de repenser le lien avec une culture dominante et avec l'extraordinaire capacité du corps social à recréer un art du difforme, d'autres façons d'être ensemble et de construire une identité à partir de l'envers des corporéités.

Contemporary representations of the body in motion can't leave the sociologist indifferent, since he tries to understand what's happening between actor and spectator. In this relation of complex interaction, what do the choreographic and circassian creations mean to the onlooker and the looked-one? Which symbolic images are at work, often contradictory but deeply efficient to reflect at once what the social inscribes on bodies, but also what appears in play expression, with the bounds, with the Other, with what goes beyond the frame?

The crossbreeding problematic is a way of rethinking the connection with a dominant culture and with the extraordinary capacity of the social body to re-create an art of the deformed, other ways of being together and building an identity from the other side of corporeities.

INDEX

Mots-clés : danse, anthropo-sociologie, métissage, art du difforme, cirque

Keywords : dance, anthropo-sociology, art of deformed, circus, cultural half-breeding

\section{AUTEURS}

BETTY LEFÈVRE

CETAPS (Centre d'Etudes des Transformations des Activités Physiques et Sportives), Faculté des Sciences du Sport. Université de Rouen

\section{MAGALI SIZORN}

CETAPS (Centre d'Etudes des Transformations des Activités Physiques et Sportives), Faculté des Sciences du Sport. Université de Rouen 